\title{
Practical Nonlinear Energy Harvesting Model in MIMO DF Relay System with Channel Uncertainty
}

\author{
Fatma Benkhelifa* and Mohamed-Slim Alouini** \\ * Imperial College London, London, UK \\ ** King Abdullah University of Science and Technology (KAUST), Thuwal, Makkah Province, Saudi Arabia \\ \{f.benkhelifa\}@imperial.ac.uk, \{slim.alouini\}@kaust.edu.sa
}

\begin{abstract}
In this paper, we aim to maximize the end-toend achievable rate of multiple-input multiple-output (MIMO) decode-and-forward (DF) where the relay is an energy harvesting (EH) node using the time switching (TS) scheme. The relay first harvests the energy from the source, then uses its harvested energy to forward the information carrying signal from the source to the destination. The $\mathrm{EH}$ model at the relay is a nonlinear model. Also, we assume that the channel knowledge is imperfect at the relay and destination. We propose the structure of the optimal covariance matrices at the source (during EH and information decoding periods), the optimal covariance matrix at the relay and the optimal EH time ratio. Through the simulation results, we compare between different linear/nonlinear EH models and we show the gain/loss performance of the linear model compared to other nonlinear EH models.
\end{abstract}

Index Terms-Multiple-input multiple-output (MIMO), decode-and-forward (DF), radio frequency energy harvesting (EH), time switching (TS), nonlinear EH model, imperfect channel state information (CSI).

\section{INTRODUCTION}

Energy harvesting $(\mathrm{EH})$ from the environment is a promising technique to make wireless communication systems selfsustainable and perpetual operable. Radio frequency (RF) signals are an emerging technique to harvest energy. The first experiences involving electromagnetic power transfer, or wireless power transfer (WPT), dates back to 1901 when Tesla tried to build the large high-voltage wireless power station, the Wardenclyffe tower that was never successful. Nowadays, WPT is getting more interest to investigate the simultaneous use of RF signals for WPT and wireless information transfer (WIT), namely the simultaneous wireless information and power transfer (SWIPT).

SWIPT has been widely studied in single-input singleoutput (SISO) systems [1], [2], in multiple-input multipleoutput (MIMO) systems [3], in MIMO relay systems [4]-[7], etc. [1] and [2] are the pioneering papers that have studied the SWIPT in SISO system through flat fading and frequency selective channels, respectively. The tradeoff region between the information rate and energy harvested, namely the R-E tradeoff region, for the co-located information decoding (ID) and $\mathrm{EH}$ receivers were investigated. Later on, [3] extended the work done in [1] and [2] to study MIMO system and considered more practical co-located receivers which are the power splitting (PS) and time switching (TS) schemes. The PS and TS schemes separate the WPT and WIT tasks over the time domain and the power domain, respectively. The
R-E tradeoff regions for the separated receivers and the colocated PS and TS practical receivers were studied for the MIMO system. In [4], a two-hop amplify-and-forward (AF) relay system was investigated where a separate multi-antenna $\mathrm{EH}$ receiver is present to harvest the energy from the source and relay data transmission using the orthogonal spacetime block codes (STBC). The rate-energy tradeoff region was characterized when the channel state information (CSI) is perfectly available and the tradeoff between the outage probability and energy were characterized when only second order statistics of CSI is available. In [5], a two-hop SISO orthogonal frequency division multiplexing (OFDM) decodeand-forward (DF) relay system was investigated where the relay harvests the energy from the source using the PS scheme. The resource allocation was studied to maximize the total achievable transmission rate. In [6], a two-hop fullduplex relay system was investigated where the relay is an EH node equipped with two antennas and using the TS scheme. Both AF and DF relaying protocols were studied, and analytical characterization of the achievable throughput was analyzed. In [7], the throughput maximization problem was considered in MIMO DF relay system with an EH relay and possibly imperfect CSI for the ideal scheme, and the practical TS and PS schemes.

However, most research works assume the linearity condition between the direct current and the stored energy. In other terms, the harvested energy is linearly dependent on the received power and the knowledge of the conversion efficiency is enough to model the EH concept. However, in practice, the $\mathrm{EH}$ concept is not linear, and the linearity assumption was always taken to simplify the analysis. Only recently, few works have proposed some nonlinear EH models either heuristic or mathematical models. In [8], a new practical nonlinear EH model was proposed and its relevance was verified through the comparison with some experimental data results. In [9], a heuristic practical nonlinear EH model was considered in studying the power allocation algorithm of MIMO Systems. In [10], a rate-energy tradeoff region of SWIPT was studied for MIMO system considering the nonlinear EH model studied in [8]. The separated EH and ID receivers and the co-located $\mathrm{EH}$ and ID receivers, namely the PS and TS schemes, were investigated. In [11], a minimization transmit power was considered where a multi-antenna hybrid access point (HAP) is transmitting data and energy to heterogeneous users where the energy receivers are using the 
PS scheme and considering a nonlinear EH model. In [12], the performance analysis of a time-slotted wireless powered communication (WPC) system was studied using a nonlinear model.

In line with this research works, we propose to maximize the end-to-end achievable rate of MIMO DF relay system where a general nonlinear EH model is assumed at the relay and imperfect CSI is assumed at the relay and the destination. The objective of this work is to check if there is a performance gain/loss in terms of the end-to-end achievable rate when we assume a nonlinear EH model.

\section{System Model}

We consider a MIMO DF relay system with a source $S$, a relay $R$ and a destination $D$. All the nodes have multiple antennas. The number of antennas at $S, R$ and $D$ are $N_{s}, N_{r}$ and $N_{d}$, respectively. The channel between $\mathrm{S}$ and $\mathrm{R}$ and the channel between $\mathrm{R}$ and $\mathrm{D}$ are denoted by $\boldsymbol{H} \in \mathbb{C}^{N_{r} \times N_{s}}$ and $\boldsymbol{G} \in \mathbb{C}^{N_{d} \times N_{r}}$, respectively, and are assumed to be quasi-static block-fading channels. We consider the half-duplex mode at the relay. The relay is an EH node that harvests the energy from the source and uses the harvested energy to forward the source signal to the destination. The relay is equipped with $\mathrm{EH}$ and ID receivers.

\section{A. Time Switching (TS) Scheme}

The relay uses the TS scheme to harvest energy. The TS receiver separates the $\mathrm{EH}$ and ID modes over two orthogonal time slots. Let $\alpha$ denote the time ratio allocated to the $\mathrm{EH}$ mode, with $0 \leq \alpha \leq 1$. Each of the transmissions from source to relay and from relay to destination occurs over a period of duration $\frac{1-\alpha}{2}$. Let $\boldsymbol{R}_{s, 1}^{T S}$ and $\boldsymbol{R}_{s, 2}^{T S}$ be the covariance matrices at the source during the ID and EH periods, respectively. Let $\boldsymbol{R}_{r}^{T S}$ be the covariance matrix at the relay. We assume that the source has an average transmit power constraint $P_{S}$, i.e., $\operatorname{tr}\left(\boldsymbol{R}_{s, i}^{T S}\right) \leq P_{s}$, for $i=1,2$, where $\operatorname{tr}(\cdot)$ denotes the trace operator and $\mathbb{E}[\cdot]$ is the mean operator.

\section{B. Nonlinear Energy Harvesting at $R$}

Usually, research works on SWIPT assume a linear EH model. Recently, few works consider more practical nonlinear EH models. In this work, we consider a general nonlinear EH model to which apply many proposed practical models in the literature. First, if $P_{S}$ is the transmit power at the source and $\boldsymbol{R}_{s, 2}^{T S}$ is the source covariance matrix during the $\mathrm{EH}$ period, the received power at the $\mathrm{EH}$ receiver is given by

$$
P_{r e c}\left(\boldsymbol{R}_{s, 2}\right)=\operatorname{tr}\left(\boldsymbol{H} \boldsymbol{R}_{s, 2} \boldsymbol{H}^{H}\right) .
$$

The harvested energy at the relay is given by

$$
Q_{r}\left(\alpha, \boldsymbol{R}_{s, 2}\right)=\alpha \Psi\left(\operatorname{tr}\left(\boldsymbol{H} \boldsymbol{R}_{s, 2} \boldsymbol{H}^{H}\right)\right),
$$

where $\Psi(\cdot)$ is the EH model either linear or not and has different modeling

$$
\Psi(x)= \begin{cases}\zeta x, & \text { if EH model is linear, } \\ \frac{\beta(x)-M \Omega}{1-\Omega}, & \text { if EH model is nonlinear 1, } \\ \xi(x) x, & \text { if EH model is nonlinear 2, }\end{cases}
$$

with $x \geq 0$ is the received power, where $\zeta \in[0,1]$ is a constant referring to conversion efficiency in the linear model, $\beta(x)=\frac{M}{1+e^{-a(x-b)}}, \Omega=\frac{1}{1+e^{a b}}, M$ is the maximum harvested energy, $a$ and $b$ are experimental parameters which reflect the nonlinear charging rate with respect to the input power and the minimum required turn-on voltage for the start of current flow through the diode [8], respectively, $\xi(x)=\frac{p_{2} x^{2}+p_{1} x+p_{0}}{q_{3} x^{3}+q_{2} x^{2}+q_{1} x+q_{0}}$ is a heuristic model for the conversion efficiency proposed in [13].

Since it is an EH model, $\Psi(x)$ should obey to a couple of properties in accordance to the law of energy conservation:

- $\Psi(x) \geq 0$.

- $\Psi(x) \leq x$.

\section{Imperfect Channel State Information at $R$ and $D$}

We assume that we have imperfect knowledge of the channel $\boldsymbol{H}$ at the relay and imperfect knowledge of the channel $\boldsymbol{G}$ at the destination.

$$
\begin{aligned}
\boldsymbol{H} & =\hat{\boldsymbol{H}}+\boldsymbol{E}_{1}+\boldsymbol{U}_{1}, \\
\boldsymbol{G} & =\hat{\boldsymbol{G}}+\boldsymbol{E}_{2}+\boldsymbol{U}_{2},
\end{aligned}
$$

where $\hat{\boldsymbol{H}}$ and $\hat{\boldsymbol{G}}$ are the estimated channels of $\boldsymbol{H}$ and $\boldsymbol{G}, \quad \boldsymbol{E}_{1}$ and $\boldsymbol{E}_{2}$ are the error matrices associated to the feedback/forward delay errors, and $\boldsymbol{U}_{1}$ and $\boldsymbol{U}_{2}$ are the error matrices associated to the channel estimation errors, respectively. $\boldsymbol{E}_{i}$ and $\boldsymbol{U}_{i}, i=1,2$, are Gaussian random matrices with zero mean and variances equal to $\sigma_{E_{i}}^{2}$ and $\sigma_{U_{i}}^{2}$, respectively. Let us denote by $\sigma_{i}^{2}=\sigma_{E_{i}}^{2}+\sigma_{U_{i}}^{2}$. The error matrices $\boldsymbol{E}_{1}$ and $\boldsymbol{U}_{1}$ and the error matrices $\boldsymbol{E}_{2}$ and $\boldsymbol{U}_{2}$ are uncorrelated with $\boldsymbol{H}$ and $\boldsymbol{G}$, respectively. We assume that $\boldsymbol{E}_{1}$ is known at the relay and $\boldsymbol{E}_{2}$ is known at the destination.

In this case, the received power at the relay becomes equal to

$$
P_{r e c}\left(\boldsymbol{R}_{s, 2}\right)=\hat{P}_{r e c}\left(\boldsymbol{R}_{s, 2}\right)+C_{1},
$$

where $\hat{P}_{r e c}\left(\boldsymbol{R}_{s, 2}\right)=\operatorname{tr}\left(\hat{\boldsymbol{H}} \boldsymbol{R}_{s, 2} \hat{\boldsymbol{H}}^{H}\right)$ and $C_{1}=r_{H} \sigma_{1}^{2} P_{s}$, where $r_{H}$ is the rank of $\boldsymbol{H}$.

Subsequently, the harvested energy and the transmit power at the relay are given by

$$
\begin{aligned}
& Q_{r}\left(\alpha, \boldsymbol{R}_{s, 2}\right)=\alpha \Psi\left(\operatorname{tr}\left(\hat{\boldsymbol{H}} \boldsymbol{R}_{s, 2} \hat{\boldsymbol{H}}^{H}\right)+C_{1}\right), \\
& P_{r}\left(\alpha, \boldsymbol{R}_{s, 2}\right)=\frac{2 \alpha}{1-\alpha} \Psi\left(\operatorname{tr}\left(\hat{\boldsymbol{H}} \boldsymbol{R}_{s, 2} \hat{\boldsymbol{H}}^{H}\right)+C_{1}\right) .
\end{aligned}
$$

Moreover, the expressions of the rates of the two hops are written as

$$
\begin{aligned}
R_{S-R}\left(\boldsymbol{R}_{s, 1}\right) & =\frac{1}{2} \log _{2}\left|\boldsymbol{I}+\frac{1}{\chi_{1}} \hat{\boldsymbol{H}} \boldsymbol{R}_{s, 1} \hat{\boldsymbol{H}}^{H}\right|, \\
R_{R-D}\left(\boldsymbol{R}_{r}\right) & =\frac{1}{2} \log _{2}\left|\boldsymbol{I}+\frac{1}{\chi_{2}\left(\alpha, \boldsymbol{R}_{s, 2}\right)} \hat{\boldsymbol{G}} \boldsymbol{R}_{r} \hat{\boldsymbol{G}}^{H}\right|,
\end{aligned}
$$

where $\chi_{1}=\sigma_{1}^{2} P_{s}+\sigma_{r}^{2}$, and $\chi_{2}\left(\alpha, \boldsymbol{R}_{s, 2}\right)=\sigma_{2}^{2} P_{r}\left(\alpha, \boldsymbol{R}_{s, 2}\right)+$ $\sigma_{d}^{2}$, where $\sigma_{r}^{2}$ and $\sigma_{d}^{2}$ are the noise variances at $\mathrm{R}$ and $\mathrm{D}$, respectively. 
The overall end-to-end rate for DF relaying protocol is given by

$$
R\left(\alpha, \boldsymbol{R}_{s, 1}, \boldsymbol{R}_{r}\right)=(1-\alpha) \min \left(R_{S-R}\left(\boldsymbol{R}_{s, 1}\right), R_{R-D}\left(\boldsymbol{R}_{r}\right)\right) .
$$

III. Rate Maximization Problem in TSR Protocol

We aim to optimize the covariance matrices at the source and the relay in a way to maximize the end-to-end achievable rate. The optimization problem can be expressed as

$$
\begin{array}{rl}
\max _{\alpha, \boldsymbol{R}_{s, 1}, \boldsymbol{R}_{s, 2}, \boldsymbol{R}_{r}} & R\left(\alpha, \boldsymbol{R}_{s, 1}, \boldsymbol{R}_{r}\right), \\
\text { s.t. } \quad & \operatorname{tr}\left(\boldsymbol{R}_{s, 1}\right) \leq P_{s}, \\
& \operatorname{tr}\left(\boldsymbol{R}_{s, 2}\right) \leq P_{s}, \\
& \operatorname{tr}\left(\boldsymbol{R}_{r}\right) \leq \frac{2 \alpha}{1-\alpha} \Psi\left(\operatorname{tr}\left(\hat{\boldsymbol{H}} \boldsymbol{R}_{s, 2} \hat{\boldsymbol{H}}^{H}\right)+C_{1}\right), \\
& \boldsymbol{R}_{s, 1} \geq 0, \boldsymbol{R}_{s, 2} \geq 0, \boldsymbol{R}_{r} \geq 0,0 \leq \alpha \leq 1,
\end{array}
$$

where $(12 b)$ and $(12 c)$ are the source transmit constraints during the ID mode and EH mode, respectively, (12d) is the transmit power constraint at the relay which is equal to the harvested energy from the source over the use duration. The rate maximization problem (12) is non-convex due to the objective function and the constraint (12d). The channel estimation errors in the R-D rate makes the objective function a non-concave function.

\section{A. New Optimization Variables}

In order to solve this problem, we first decouple the dependence of the R-D rate on $\boldsymbol{R}_{s, 2}$ and $\boldsymbol{R}_{r}$, by using other optimization variables which are

$$
\begin{aligned}
\tilde{\boldsymbol{R}}_{s, 1} & =\frac{1}{\chi_{1}} \boldsymbol{R}_{s, 1}, \\
\tilde{\boldsymbol{R}}_{r} & =\frac{1}{\sigma_{2}^{2} \frac{2 \alpha}{1-\alpha} \Psi\left(\operatorname{tr}\left(\hat{\boldsymbol{H}} \boldsymbol{R}_{s, 2} \hat{\boldsymbol{H}}^{H}\right)+C_{1}\right)+\sigma_{d}^{2}} \boldsymbol{R}_{r} .
\end{aligned}
$$

We denote by $\Phi(\cdot, \cdot)$ the function defined as:

$$
\Phi(\alpha, x)=\frac{\frac{2 \alpha}{1-\alpha} \Psi\left(x+C_{1}\right)}{\sigma_{2}^{2} \frac{2 \alpha}{1-\alpha} \Psi\left(x+C_{1}\right)+\sigma_{d}^{2}} .
$$

Using these new optimization variables, the optimization problem becomes

$$
\begin{aligned}
\max _{\alpha, \tilde{\boldsymbol{R}}_{s, 1}, \boldsymbol{R}_{s, 2}, \tilde{\boldsymbol{R}}_{r}} & \frac{1-\alpha}{2} \min \left(\log _{2}\left|\boldsymbol{I}+\hat{\boldsymbol{H}} \tilde{\boldsymbol{R}}_{s, 1} \hat{\boldsymbol{H}}^{H}\right|,\right. \\
& \left.\log _{2}\left|\boldsymbol{I}+\hat{\boldsymbol{G}}_{\tilde{\boldsymbol{R}}_{r}} \hat{\boldsymbol{G}}^{H}\right|\right), \\
\text { s.t. } & \operatorname{tr}\left(\tilde{\boldsymbol{R}}_{s, 1}\right) \leq \frac{P_{s}}{\chi_{1}}, \\
& \operatorname{tr}\left(\boldsymbol{R}_{s, 2}\right) \leq P_{s}, \\
& \operatorname{tr}\left(\tilde{\boldsymbol{R}}_{r}\right) \leq \Phi\left(\alpha, \operatorname{tr}\left(\hat{\boldsymbol{H}} \boldsymbol{R}_{s, 2} \hat{\boldsymbol{H}}^{H}\right)\right), \\
& \tilde{\boldsymbol{R}}_{s, 1} \geq 0, \boldsymbol{R}_{s, 2} \geq 0, \tilde{\boldsymbol{R}}_{r} \geq 0,0 \leq \alpha \leq 1 .
\end{aligned}
$$

This step solves the non-convexity problem of the objective function. But, still we cannot solve this problem due to the right-hand side of the constraint (16d). Next, in order to solve the obtained problem, we first fix $\alpha \in(0,1)$ and we solve for a given $\alpha$. Then, the optimal $\alpha$ can be obtained using any one-dimensional search method such as the greedy search or the bisection method. Note that it is clear why the optimal $\alpha$ cannot be zero or one. Because, in both cases, the overall rate will be zero. Next, we solve for a given $\alpha \in(0,1)$.

\section{B. Properties of $\Phi$}

In what follows, we present the following lemma regarding the function $\Phi$ :

Lemma 1: $\Phi(\alpha, x)$ is a monotonically increasing function with respect to $x$, if $\Psi(x)$ does so, and is a monotonically increasing function with respect to $\alpha$.

Proof: The proof can be easily obtained by just deriving the expression of $\Phi(\alpha, x)$. The derivative of $\Phi(\alpha, x)$ with respect to $x$ can be expressed as

$$
\frac{\delta \Phi(\alpha, x)}{\delta x}=\frac{\frac{2 \alpha}{1-\alpha} \sigma_{d}^{2} \frac{\delta \Psi\left(x+C_{1}\right)}{\delta x}}{\left(\sigma_{2}^{2} \frac{2 \alpha}{1-\alpha} \Psi\left(x+C_{1}\right)+\sigma_{d}^{2}\right)^{2}} .
$$

So, $\Phi(\alpha, x)$ is a monotonically increasing function with respect to $x$, if $\Psi(x)$ does also. Moreover, the derivative of $\Phi(\alpha, x)$ with respect to $\alpha$ is expressed as

$$
\frac{\delta \Phi(\alpha, x)}{\delta \alpha}=\frac{2 \Psi\left(x+C_{1}\right) \sigma_{d}^{2}}{(1-\alpha)^{2}\left(\sigma_{2}^{2} \frac{2 \alpha}{1-\alpha} \Psi\left(x+C_{1}\right)+\sigma_{d}^{2}\right)^{2}}>0 .
$$

So, $\Phi(\alpha, x)$ is a monotonically increasing function with respect to $\alpha$.

This result is important as it will allow us to simplify our optimization problem.

Remark 1: Note that the function $\Psi(\cdot)$ as defined in (3) is a monotonically increasing function. If another EH model is considered, Lemma 1 holds as long as the new function $\Psi(\cdot)$ is a monotonically increasing function. Also, the condition in Lemma 1 makes sense in the way that the more the power is received, the more the energy is harvested. Next, we solve the optimization problem (16) for a given $\alpha \in(0,1)$.

\section{Optimal Source Covariance Matrix During EH Mode $\boldsymbol{R}_{s, 2}$}

At this point, we can see that the source covariance matrix $\boldsymbol{R}_{s, 2}$ during the $\mathrm{EH}$ mode is only present in the constraints (16c), (16d), and (16e).

Lemma 2: The optimal source covariance matrix $\boldsymbol{R}_{s, 2}$ during the $\mathrm{EH}$ mode is the one that maximizes the right hand side of the constraint (16d), which is the harvested energy at the relay during the EH period, as long as (16c) is satisfied.

Proof: We can show this observation by contradiction. If the triplet $\tilde{\boldsymbol{R}}_{s, 1}^{*}, \boldsymbol{R}_{s, 2}^{*}$, and $\tilde{\boldsymbol{R}}_{r}^{*}$ are the optimal solution to our optimization problem (16) such that $\Phi\left(\alpha, \operatorname{tr}\left(\hat{\boldsymbol{H}} \boldsymbol{R}_{s, 2}^{*} \hat{\boldsymbol{H}}^{H}\right)\right)<$ $\max _{\text {s.t.tr }\left(\boldsymbol{R}_{s, 2} \leq P_{s}\right.} \Phi\left(\alpha, \operatorname{tr}\left(\hat{\boldsymbol{H}} \boldsymbol{R}_{s, 2} \hat{\boldsymbol{H}}^{H}\right)\right)$. So, there exists another source covariance matrix $\hat{\boldsymbol{R}}_{s, 2}$ s.t. $\Phi\left(\alpha, \operatorname{tr}\left(\hat{\boldsymbol{H}} \hat{\boldsymbol{R}}_{s, 2} \hat{\boldsymbol{H}}^{H}\right)\right)>$ $\Phi\left(\alpha, \operatorname{tr}\left(\hat{\boldsymbol{H}} \boldsymbol{R}_{s, 2}^{*} \hat{\boldsymbol{H}}^{H}\right)\right)$. Thus, the overall rate of the triplet $\tilde{\boldsymbol{R}}_{s, 1}^{*}$, $\hat{\boldsymbol{R}}_{s, 2}$, and $\tilde{\boldsymbol{R}}_{r}^{*}$ will be greater than the overall rate of the optimal solution, which is in contradiction. 
So, it is clear that in order to maximize the overall end-to-end rate, the optimal $\boldsymbol{R}_{s, 2}$ is the one that maximizes right hand side of the constraint (16d), which is the harvested energy at the relay during the $\mathrm{EH}$ period.

Subsequently, the optimal source covariance matrix $\boldsymbol{R}_{s, 2}$ is given in the following corollary

Corollary 1: Using Lemma 2, the optimal source covariance matrix $\boldsymbol{R}_{s, 2}$ is written as

$$
\boldsymbol{R}_{s, 2}^{*}=P_{s} \boldsymbol{v}_{\hat{H}, 1} \boldsymbol{v}_{\hat{H}, 1}^{H},
$$

where $\boldsymbol{v}_{\hat{H}, 1}$ is the eigenvector corresponding to the maximum eigenvalue $\lambda_{\hat{H}, 1}$ of $\hat{\boldsymbol{H}}^{H} \hat{\boldsymbol{H}}$.

Proof: Using Lemma 2, the optimal source covariance matrix $\boldsymbol{R}_{s, 2}$ is solution to

$$
\begin{aligned}
\max _{\boldsymbol{R}_{s, 2}} & \Phi\left(\alpha, \operatorname{tr}\left(\hat{\boldsymbol{H}} \boldsymbol{R}_{s, 2} \hat{\boldsymbol{H}}^{H}\right)\right), \\
\text { s.t. } & \operatorname{tr}\left(\boldsymbol{R}_{s, 2}\right) \leq P_{s}, \\
& \boldsymbol{R}_{s, 2} \geq 0 .
\end{aligned}
$$

Subsequently, as was shown in Lemma 2 in [10], the solution of the energy harvested maximization problem is equivalent to the received power maximization problem as long as the function $\Phi(\alpha, \cdot)$ is a monotonically increasing with respect to $x$. So, the optimal source covariance matrix $\boldsymbol{R}_{s, 2}$ is also solution to

$$
\begin{array}{cl}
\max _{\boldsymbol{R}_{s, 2}} & \operatorname{tr}\left(\hat{\boldsymbol{H}} \boldsymbol{R}_{s, 2} \hat{\boldsymbol{H}}^{H}\right), \\
\text { s.t. } & \operatorname{tr}\left(\boldsymbol{R}_{s, 2}\right) \leq P_{s}, \\
& \boldsymbol{R}_{s, 2} \geq 0 .
\end{array}
$$

The obtained equivalent problem is well-investigated in SWIPT research works and its solution is ranked one and is given by (19).

Using Corollary 1, the received power, the harvested energy, and $\Phi\left(\alpha, \operatorname{tr}\left(\hat{\boldsymbol{H}} \boldsymbol{R}_{s, 2} \hat{\boldsymbol{H}}^{H}\right)\right)$ are given by

$$
\begin{aligned}
P_{\text {rec }, \text { max }} & =P_{s} \lambda_{\hat{H}, 1}+C_{1}, \\
\Psi_{\text {max }} & =\Psi\left(P_{s} \lambda_{\hat{H}, 1}+C_{1}\right), \\
Q_{r, \text { max }}(\alpha) & =\alpha \Psi_{\text {max }}, \\
\Phi_{\text {max }}(\alpha) & =\Phi\left(\alpha, \operatorname{tr}\left(\hat{\boldsymbol{H}} \boldsymbol{R}_{s, 2}^{*} \hat{\boldsymbol{H}}^{H}\right)\right)=\frac{\frac{2 \alpha}{1-\alpha} \Psi_{\max }}{\sigma_{2}^{2} \frac{2 \alpha}{1-\alpha} \Psi_{\max }+\sigma_{d}^{2}},
\end{aligned}
$$

respectively.

\section{Optimal Source and Relay Covariance Matrices During ID Mode $\boldsymbol{R}_{s, 1}$ and $\boldsymbol{R}_{r}$}

First, let us consider the singular value decompositions (SVDs) of $\hat{\boldsymbol{H}}$ and $\hat{\boldsymbol{G}}$ are

$$
\begin{aligned}
& \hat{\boldsymbol{H}}=\boldsymbol{U}_{\hat{H}} \boldsymbol{D}_{\hat{H}}^{1 / 2}\left(\boldsymbol{V}_{\hat{H}}\right)^{H}, \\
& \hat{\boldsymbol{G}}=\boldsymbol{U}_{\hat{G}} \boldsymbol{D}_{\hat{G}}^{1 / 2}\left(\boldsymbol{V}_{\hat{G}}\right)^{H},
\end{aligned}
$$

respectively, where $\boldsymbol{U}_{\hat{H}}, \boldsymbol{V}_{\hat{H}}, \boldsymbol{U}_{\hat{G}}$ and $\boldsymbol{V}_{\hat{G}}$ are unitary matrices with dimensions $N_{r} \times r_{H}, N_{s} \times r_{H}, N_{d} \times r_{G}$ and $N_{r} \times r_{G}$, respectively, $\boldsymbol{D}_{\hat{H}}=\operatorname{diag}\left(\lambda_{\hat{H}, 1}, \ldots, \lambda_{\hat{H}, r_{H}}\right)$ and $\boldsymbol{D}_{\hat{G}}=$ $\operatorname{diag}\left(\lambda_{\hat{G}, 1}, \ldots, \lambda_{\hat{G}, r_{G}}\right)$ are the diagonal matrices containing the eigenvalues arranged in a decreasing order of $\hat{\boldsymbol{H}} \hat{\boldsymbol{H}}^{H}$ and $\hat{\boldsymbol{G}} \hat{\boldsymbol{G}}^{H}$, respectively, and $r_{H}$ and $r_{G}$ are the rank of $\hat{\boldsymbol{H}}$ and $\hat{\boldsymbol{G}}$, respectively.

The optimal source and relay covariance matrices during ID mode $\boldsymbol{R}_{s, 1}$ and $\boldsymbol{R}_{r}$ are expressed in the following corollary

Corollary 2: The optimal source and relay covariance matrices during ID mode $\boldsymbol{R}_{s, 1}$ and $\boldsymbol{R}_{r}$ are expressed as

$$
\begin{aligned}
\boldsymbol{R}_{s, 1}^{*} & =\chi_{1} \boldsymbol{V}_{\hat{H}} \tilde{\boldsymbol{D}}_{s} \boldsymbol{V}_{\hat{H}}^{H}, \\
\boldsymbol{R}_{r}^{*} & =\left(\sigma_{2}^{2} \frac{2 \alpha}{1-\alpha} \Psi_{\text {max }}+\sigma_{d}^{2}\right) \boldsymbol{V}_{\hat{G}} \tilde{\boldsymbol{D}}_{r} \boldsymbol{V}_{\hat{G}}^{H},
\end{aligned}
$$

where $\tilde{\boldsymbol{D}}_{s}=\operatorname{diag}\left(\tilde{\lambda}_{s, 1}, \ldots, \tilde{\lambda}_{s, r_{H}}\right)$ and $\tilde{\boldsymbol{D}}_{r}=$ $\operatorname{diag}\left(\tilde{\lambda}_{r, 1}, \ldots, \tilde{\lambda}_{r, r_{G}}\right)$ are the diagonal matrices whose elements are given by the water-filling solution [14] as $\tilde{\lambda}_{s, i}=\left(\frac{1}{\beta_{1}}-\frac{1}{\lambda_{\hat{H}, i}}\right)^{+}$, for $i=1, \ldots, r_{H}$, and $\tilde{\lambda}_{r, j}=\left(\frac{1}{\beta_{2}}-\frac{1}{\lambda_{\hat{G}, j}}\right)^{+}$, for $j=1, \ldots, r_{G}$, and $\beta_{1}$ and $\beta_{2}$ are the Lagrange multiplier satisfying the constraints $\sum_{i=1}^{r_{H}} \tilde{\lambda}_{s, i}=\frac{P_{s}}{\chi_{1}}$, and $\sum_{j=1}^{r_{G}} \tilde{\lambda}_{r, j}=\Phi_{\max }(\alpha)$, respectively.

Proof: Applying the results in Corollary 1 to the optimization problem (16), we obtain the following optimization problem which optimizes the covariance matrices $\tilde{\boldsymbol{R}}_{s, 1}$ and $\tilde{\boldsymbol{R}}_{r}$ as

$$
\begin{aligned}
\max _{\tilde{\boldsymbol{R}}_{s, 1}, \tilde{\boldsymbol{R}}_{r}} & \min \left(\log _{2}\left|\boldsymbol{I}+\hat{\boldsymbol{H}} \tilde{\boldsymbol{R}}_{s, 1} \hat{\boldsymbol{H}}^{H}\right|, \log _{2}\left|\boldsymbol{I}+\hat{\boldsymbol{G}} \tilde{\boldsymbol{R}}_{r} \hat{\boldsymbol{G}}^{H}\right|\right) \\
\text { s.t. } & \operatorname{tr}\left(\tilde{\boldsymbol{R}}_{s, 1}\right) \leq \frac{P_{s}}{\chi_{1}} \\
& \operatorname{tr}\left(\tilde{\boldsymbol{R}}_{r}\right) \leq \Phi_{\max }(\alpha) \\
& \tilde{\boldsymbol{R}}_{s, 1} \geq 0, \tilde{\boldsymbol{R}}_{r} \geq 0
\end{aligned}
$$

The problem (30) is convex and the constraints are decoupled. We can split this optimization problem into two subproblems which are

$$
\begin{array}{cl}
\max _{\tilde{\boldsymbol{R}}_{s, 1}} & \log _{2}\left|\boldsymbol{I}+\hat{\boldsymbol{H}} \tilde{\boldsymbol{R}}_{s, 1} \hat{\boldsymbol{H}}^{H}\right|, \\
\text { s.t. } & \operatorname{tr}\left(\tilde{\boldsymbol{R}}_{s, 1}\right) \leq \frac{P_{s}}{\chi_{1}}, \\
& \tilde{\boldsymbol{R}}_{s, 1} \geq 0, \\
\max _{\tilde{\boldsymbol{R}}_{r}} & \log _{2}\left|\boldsymbol{I}+\hat{\boldsymbol{G}} \tilde{\boldsymbol{R}}_{r} \hat{\boldsymbol{G}}^{H}\right|, \\
\text { s.t. } & \operatorname{tr}\left(\tilde{\boldsymbol{R}}_{r}\right) \leq \Phi_{\max }(\alpha), \\
& \tilde{\boldsymbol{R}}_{r} \geq 0 .
\end{array}
$$

These two subproblems are equivalent to the throughput maximization of one-hop MIMO system given an average transmit power constraint at the transmitter in (31b) and (31e) and their optimal solution $\tilde{\boldsymbol{R}}_{s, 1}$ and $\tilde{\boldsymbol{R}}_{r}$ have the following forms

$$
\begin{aligned}
\tilde{\boldsymbol{R}}_{s, 1} & =\boldsymbol{V}_{\hat{H}} \tilde{\boldsymbol{D}}_{s} \boldsymbol{V}_{\hat{H}}^{H}, \\
\tilde{\boldsymbol{R}}_{r} & =\boldsymbol{V}_{\hat{G}} \tilde{\boldsymbol{D}}_{r} \boldsymbol{V}_{\hat{G}}^{H} .
\end{aligned}
$$

Substituting these expressions in (13) and (14), we get the results in the corollary 2 
Subsequently, the corresponding achievable rates over the S-R link and the R-D link are given by

$$
\begin{gathered}
R_{S-R}\left(\boldsymbol{R}_{s, 1}\right)=\sum_{i=1}^{r_{H}} \log _{2}\left(1+\lambda_{\hat{H}, i} \tilde{\lambda}_{s, i}\right), \\
R_{R-D}\left(\boldsymbol{R}_{r}\right)=\sum_{i=1}^{r_{G}} \log _{2}\left(1+\lambda_{\hat{G}, i} \tilde{\lambda}_{r, i}\right) .
\end{gathered}
$$

\section{E. Optimal EH Time Ratio $\alpha$}

The optimal time ratio for the $\mathrm{EH}$ slot $\alpha$ is given in the following corollary:

Corollary 3: The optimal time ratio for the EH slot $\alpha$ is expressed as

$$
\alpha=\frac{S \sigma_{d}^{2}}{2 \Psi_{\text {max }}-S\left(2 \sigma_{2}^{2} \Psi_{\text {max }}-\sigma_{d}^{2}\right)} .
$$

Proof: Using the previous results, the equivalent optimization problem with respect to $\alpha$ is

$$
\begin{aligned}
\max _{\alpha} & (1-\alpha) \sum_{i=1}^{r_{G}} \log _{2}\left(1+\lambda_{\hat{G}, i} \tilde{\lambda}_{r, i}\right), \\
\text { s.t. } & \sum_{j=1}^{r_{G}} \tilde{\lambda}_{r, j} \leq \frac{\frac{2 \alpha}{1-\alpha} \Psi_{\max }}{\sigma_{2}^{2} \frac{2 \alpha}{1-\alpha} \Psi_{\max }+\sigma_{d}^{2}}, \\
& 0<\alpha<1 .
\end{aligned}
$$

If the constraint (37b) is inactive, the optimal $\alpha$ will converge to zero. Otherwise, the optimal $\alpha$ is the one that verifies the constraint (37b) with equality.

$$
\operatorname{tr}\left(\tilde{\boldsymbol{R}}_{r}\right)=S=\sum_{j=1}^{r_{G}} \tilde{\lambda}_{r, j}=\frac{\frac{2 \alpha}{1-\alpha} \Psi_{\max }}{\sigma_{2}^{2} \frac{2 \alpha}{1-\alpha} \Psi_{\max }+\sigma_{d}^{2}} .
$$

Hence, the optimal $\alpha$ is given by

$$
\alpha=\frac{S \sigma_{d}^{2}}{2 \Psi_{\max }-S\left(2 \sigma_{2}^{2} \Psi_{\max }-\sigma_{d}^{2}\right)} .
$$

\section{Numerical Results}

In this section, we present some numerical results to show the maximum achievable rate of MIMO DF relay system with different linear and nonlinear EH models and with possibly imperfect CSI. For the nonlinear EH models, we are using the nonlinear EH model based on the sigmoidal function proposed in [8], and the heuristic model for the conversion efficiency in [13]. To make sure that Lemma 1 is satisfied, we choose the EH models with an increasing function $\Psi(\cdot)$. The nonlinear models are based on two experimental results from [15] and [16]. First, the data measurements in [15] were shown in [8], [10] to be fitted by the sigmoidal model with parameters $a=1500, b=0.0022$, and $M=24 \mathrm{~mW}$. Moreover, the data measurements in [16] were shown to be fitted by the sigmoidal model with parameters with parameters $a=47083, b=2.9 \mathrm{uW}$, and $M=24 \mathrm{~mW}$ in [12], and by the heuristic function with parameters $p_{2}=1.23, p_{1}=10.5, p_{0}=-0.238, q_{3}=1$, $q_{2}=-0.125, q_{1}=0.24$, and $q_{0}=0.00045$ in [13]. Also, the
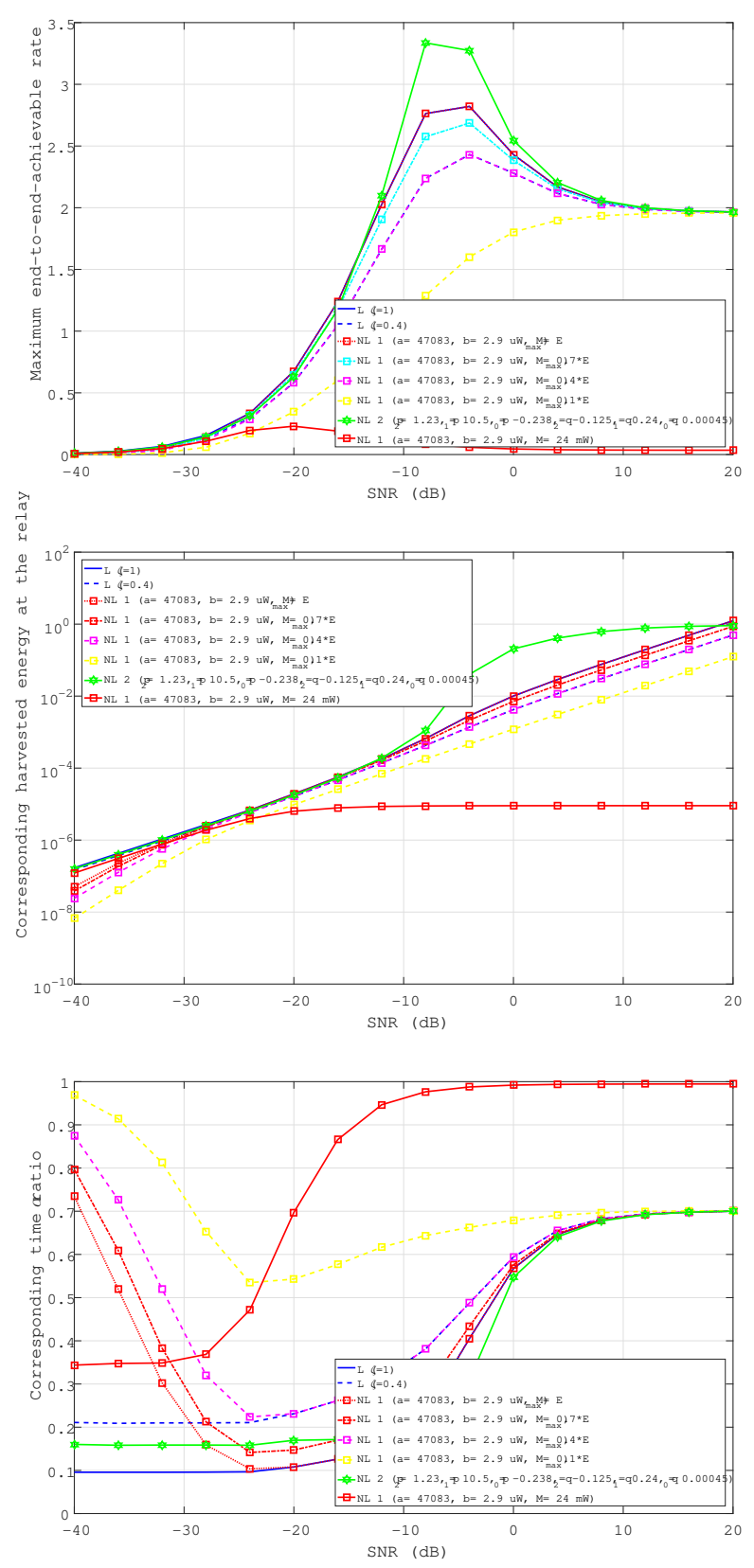

Figure 1. The achievable end-to-end rate, the corresponding harvested energy at $\mathrm{R}$, and the corresponding time ratio $\alpha$ versus the transmit power $P_{s}$ at $\mathrm{S}$ in $\mathrm{dB}$, with $N_{s}=N_{r}=N_{d}=4, \sigma_{r}^{2}=\sigma_{d}^{2}=10^{-4} \mathrm{~dB}$, with imperfect CSI $\sigma_{E_{i}}^{2}=\sigma_{U_{i}}^{2}=0.1$ and the nonlinear models in agreement with [16].

authors in [13] showed that the data in [17] has a maximum conversion efficiency $60 \%$. So, the linear model is considered with two conversion efficiencies: the maximum one $\zeta=1$ and a more realistic one $\zeta=0.4$. The number of transmit antennas at all the nodes is equal to 4 and the noise variances at the relay and destination are equal to $-40 \mathrm{~dB}$ [9]. In all figures, the value of $E_{\max }$ corresponds to maximum harvested energy $\Psi_{\max }$ varying with the EH model considered either linear or nonlinear (sigmoidal or heuristic).

In Fig. 1, we have plotted the achievable end-to-end 
rate, the corresponding harvested energy at $\mathrm{R}$, and the corresponding time ratio $\alpha$ versus the transmit power $P_{s}$ at $\mathrm{S}$ in $\mathrm{dB}$, with imperfect CSI $\sigma_{E_{i}}^{2}=\sigma_{U_{i}}^{2}=0.1$. The nonlinear $\mathrm{EH}$ models are following the data measurements in [16] with parameters $a=47083, b=2.9 \mathrm{uW}$, and $M=24 \mathrm{~mW}$ [12] for the sigmoidal nonlinear EH model, and $p_{2}=1.23, p_{1}=10.5, p_{0}=-0.238, q_{3}=1, q_{2}=-0.125$, $q_{1}=0.24$, and $q_{0}=0.00045$ [13] for the heuristic nonlinear EH model. First, we can see that the heuristic nonlinear EH model outperforms all the other linear/nonlinear EH models in terms of the maximum end-to-end rate. Moreover, the sigmoidal nonlinear $\mathrm{EH}$ model with $M=E_{\max }$ and $M=0.7 E_{\max }$ outperforms the linear model with a practical conversion efficiency $\zeta=0.4$, for $-10<S N R \leq 10 \mathrm{~dB}$. Otherwise, all the EH models perform alike in terms of the maximum end-to-end rate. In terms of the harvested energy, the relay harvests more energy for the heuristic nonlinear $\mathrm{EH}$ model, especially in the high power regime. We can see also that the linear models with both $\zeta=1$ and $\zeta=0.4$ and the heuristic nonlinear EH model have the lowest time ratio $\alpha$, especially in the low power regime. At this point, we can say that the heuristic nonlinear EH model presents a performance gain in terms of end-to-end rate, especially in the high power regime.

In Fig. 2, we have plotted the achievable end-to-end rate, the corresponding harvested energy at $\mathrm{R}$, and the corresponding time ratio $\alpha$ versus the transmit power $P_{s}$ at $\mathrm{S}$ in $\mathrm{dB}$, with imperfect CSI $\sigma_{E_{i}}^{2}=\sigma_{U_{i}}^{2}=0.1$. The nonlinear EH models are following the data measurements in [15] with parameters $a=1500$, and $b=0.0022$. First, we can see that the sigmoidal nonlinear EH model proposed in [8], [10] with parameters $a=1500, b=0.0022$, and $M=24 \mathrm{~mW}$ achieves the highest maximum end-to-end rate and outperforms the linear model with the maximum conversion efficiency $\zeta=1$ and all the other EH models. Moreover, we can see that the sigmoidal nonlinear EH model with $M=E_{\max }$ and $M=0.7 E_{\max }$ outperforms the linear model with a practical conversion efficiency $\zeta=0.4$ in the high power regime. However, in the low power regime, the linear models with both $\zeta=1$ and $\zeta=0.4$ outperform all the nonlinear models except the one with $M=24 \mathrm{~mW}$. At this point, we can see that the linear model engenders losses in terms of end-to-end rate, in the high power regime. While, the sigmoidal nonlinear EH model with $M=24 \mathrm{~mW}$ presents a performance gain compared to the other schemes, in terms of end-to-end rate. In terms of the harvested energy, the relay harvests more energy for the linear models with both $\zeta=1$ and $\zeta=0.4$ and the sigmoidal nonlinear EH model with $M=24 \mathrm{~mW}$. We can see also that the time ratio $\alpha$ for the linear models with both $\zeta=1$ and $\zeta=0.4$ and the sigmoidal nonlinear $\mathrm{EH}$ model with $M=24 \mathrm{~mW}$ is the lowest one, especially in the low power regime.

\section{Conclusion}

In this paper, we have studied the rate maximization problem for the MIMO DF relay system where the relay is an EH node using a nonlinear EH model and with imperfect


Figure 2. The achievable end-to-end rate, the corresponding harvested energy at $\mathrm{R}$, and the corresponding time ratio $\alpha$ versus the transmit power $P_{s}$ at $\mathrm{S}$ in $\mathrm{dB}$, with $N_{s}=N_{r}=N_{d}=4, \sigma_{r}^{2}=\sigma_{d}^{2}=10^{-4} \mathrm{~dB}$, with imperfect CSI $\sigma_{E_{i}}^{2}=\sigma_{U_{i}}^{2}=0.1$ and the nonlinear models in agreement with [15].

CSI at the relay and the destination. We have proposed the source and relay covariance matrices and the optimal time ratio $\alpha$ for the $\mathrm{EH}$ mode. Through the simulation results, we compared between different linear/nonlinear EH models and we have highlighted the gain/loss performance when using the linear EH model compared to the other nonlinear EH model. We have seen that the linear model has the same performance as the nonlinear models for very low power regime. For the high power regime, either the heuristic or the sigmoidal nonlinear EH model outperforms the linear 
EH model depending on the circuitry used.

\section{REFERENCES}

[1] L. Varshney, "Transporting information and energy simultaneously," in IEEE International Symposium on Information Theory (ISIT'2008), July 2008, pp. 1612-1616.

[2] P. Grover and A. Sahai, "Shannon meets Tesla: Wireless information and power transfer," in IEEE International Symposium on Information Theory Proceedings (ISIT'2010), June 2010, pp. 2363-2367.

[3] R. Zhang and C. K. Ho, "MIMO broadcasting for simultaneous wireless information and power transfer," IEEE Transactions on Wireless Communications, vol. 12, no. 5, pp. 1989-2001, May 2013.

[4] B. Chalise, W.-K. Ma, Y. Zhang, H. Suraweera, and M. Amin, "Optimum performance boundaries of OSTBC based AF-MIMO relay system with energy harvesting receiver," IEEE Transactions on Signal Processing, vol. 61, no. 17, pp. 4199-4213, September 2013.

[5] X. Di, K. Xiong, and Z. Qiu, "Simultaneous wireless information and power transfer for two-hop OFDM relay system," CoRR, vol. abs/1407.0166, 2014. [Online]. Available: http://arxiv.org/abs/1407.0166

[6] C. Zhong, H. Suraweera, G. Zheng, I. Krikidis, and Z. Zhang, "Wireless information and power transfer with full duplex relaying," IEEE Transactions on Communications, vol. 62, no. 10, pp. $3447-$ 3461, October 2014.

[7] F. Benkhelifa, A. S. Salem, and M. S. Alouini, "Rate maximization in MIMO Decode-and-Forward communications with an $\mathrm{EH}$ relay and possibly imperfect CSI," IEEE Transactions on Communications, vol. 64, no. 11, pp. 4534-4549, November 2016.

[8] E. Boshkovska, D. W. K. Ng, N. Zlatanov, and R. Schober, "Practical non-linear energy harvesting model and resource allocation for SWIPT systems," IEEE Communications Letters, vol. 19, no. 12, pp. 2082 2085, December 2015
[9] L. Shi, L. Zhao, and K. Liang, "Power allocation for wireless powered MIMO transmissions with non-linear RF energy conversion models," China Communications, vol. 14, no. 2, pp. 57-64, February 2017.

[10] K. Xiong, B. Wang, and K. J. R. Liu, "Rate-energy region of SWIPT for MIMO broadcasting under nonlinear energy harvesting model," IEEE Transactions on Wireless Communications, vol. 16, no. 8, pp. 5147-5161, August 2017.

[11] R. Jiang, K. Xiong, P. Fan, Y. Zhang, and Z. Zhong, "Optimal design of SWIPT systems with multiple heterogeneous users under non-linear energy harvesting model," IEEE Access, vol. 5, pp. 11479-11489, 2017.

[12] R. Morsi, E. Boshkovska, E. Ramadan, D. W. K. Ng, and R. Schober, "On the performance of wireless powered communication with non-linear energy harvesting," CoRR, vol. abs/1702.07881, 2017. [Online]. Available: http://arxiv.org/abs/1702.07881

[13] Y. Chen, K. T. Sabnis, and R. A. Abd-Alhameed, "New formula for conversion efficiency of RF EH and its wireless applications," IEEE Transactions on Vehicular Technology, vol. 65, no. 11, pp. 9410-9414, November 2016.

[14] T. M. Cover and J. A. Thomas, Elements of Information Theory. John Wiley and Sons Inc., 1991.

[15] J. Guo and X. Zhu, "An improved analytical model for RF-DC conversion efficiency in microwave rectifiers," in 2012 IEEE/MTT-S International Microwave Symposium Digest, June 2012, pp. 1-3.

[16] M. Stoopman, S. Keyrouz, H. J. Visser, K. Philips, and W. A. Serdijn, "A self-calibrating RF energy harvester generating $1 \mathrm{~V}$ at $-26.3 \mathrm{dBm}$," in 2013 Symposium on VLSI Circuits, June 2013, pp. C226-C227.

[17] T. Le, K. Mayaram, and T. Fiez, "Efficient far-field radio frequency energy harvesting for passively powered sensor networks," IEEE Journal of Solid-State Circuits, vol. 43, no. 5, pp. 1287-1302, May 2008 